\title{
BER Enhancement of MC-CDMA through B-STTC Based STBC and STBC Based B-STTC Site Diversity Techniques
}

\author{
M.Pushpakodi ${ }^{1}$, N. Kumaratharan,"* \\ ${ }^{1}$ Department of Electronics and Communication Engineering, Cahcet, Melvisharam, India \\ ${ }^{2}$ Department of Information Technology, Sri Venkateswara College of Engineering, Irungatukottai - 602 117, Chennai, India \\ *Corresponding author: kumaratharan@rediffmail.com
}

Copyright $(2013$ Horizon Research Publishing All rights reserved.

\begin{abstract}
The combination of multiple antennas and multi-carrier code division multiple access (MC-CDMA) is a strong candidate for the downlink of future mobile communications. The advancement of such systems, in scenarios that model real life transmissions is an additional step towards an optimised achievement. Nevertheless, when transmitting over fading channel multi-cell interference occurs and this degrades the performance of the system. Site diversity technique is applied to the system to overcome multi-cell interference. Due to non orthogonality of spreading codes multi-cell interference is not completely eradicated. To overcome this problem, space time block code (STBC) based space time trellis code (STTC) site diversity technique was introduced to reduce multi-cell interference. In this paper, balanced STTC (B-STTC) based STBC site diversity is proposed to further improve the performance of MC-CDMA system by mitigating multi-cell interference and is extended to STBC based B-STTC site diversity technique. Simulation result shows that STBC based B-STTC site diversity outperforms B-STTC based STBC site diversity technique.
\end{abstract}

Keywords MC-CDMA, STBC, STTC, B-STTC

\section{Introduction}

Broadband wireless access for evolving mobile internet and multimedia services are driving a surge of research on future wireless communication systems to support multi-user access and high data rates. Multi-carrier code division multiple access (MC-CDMA), which suits high data rate applications with multiplexing technique appears to be a promising technique in achieving high data rates [1]. MC-CDMA is robust to multi-path fading, inheriting the advantages of conventional CDMA where frequency diversity can be achieved in a broadband channel [2]. With its capability of synchronous transmission, MC-CDMA is suitable for downlink of cellular communication systems [3]. The challenge of achieving reliable data transmission over wireless link is more difficult due to the fact that received signals from multi-paths add destructively, which results in serious performance degradation. To achieve reliable communication over wireless links antenna diversity was introduced into the system by employing spatially separated antennas at the transmitter and receiver [4]. The advantage of using multiple antennas is to increase the channel capacity [4]. High data rate MC-CDMA systems additionally employs multiple input multiple output (MIMO) techniques, e.g., Alamouti codes and space time block codes (STBC) to mitigate fading [5].

Data transmission involves spreading operations by the use of short channelisation code and long scrambling code. Short channelisation code helps in separating the signals of different users present within the cell and long scrambling code mitigates the effects of interference produced by users belonging to other cells. However, the system faces multi-cell interference due to fading channel resulting in degradation of bit-error rate (BER).

Site diversity technique has been proposed for realizing CDMA and orthogonal frequency division multiplexing (OFDM) systems to minimize multi-cell interference [5-7], where STBC is used to gain diversity effect among several base stations. STBC site diversity system transmits the encoded signals from several base stations and these signals are combined at the receiver with STBC decoding operation. STBC branches and the scrambling codes are assigned to each base station to maintain orthogonality of signals between the cells and to reduce interference among them. The same technique is extended to MC-CDMA system. However, the scrambling codes assigned are generally non orthogonal among cells and hence multi-cell interference still exists. Using STBC with multiple antennas at each base station, site diversity was achieved with further reduction in multi-cell interference [8]. STBC does not provide coding 
gain and in view of this it is worthwhile to consider a joint design of error control coding, modulation, transmit and receive diversity to develop an effective signalling scheme called space time trellis code (STTC), which combats the effects of fading [9]. STTC became extremely popular as it can simultaneously offer coding gain with spectral efficiency and full diversity over fading channels. STTC was used to obtain site diversity with multiple antennas at base station and it outperformed STBC based site diversity in terms of error rates [10]. A new class of 4-phase shift keying (4-PSK) STTC using points of constellation with same probability called as balanced STTC (B-STTC) [11] was proposed. B-STTC with equal probable of constellation points achieves reduced error rate [11] and it is used to achieve site diversity for MC-CDMA system [12] to enhance its performance. STBC based STTC codes [13] built with set partitioning [14], achieves more diversity gain and achieves better performance in terms of error rates, was used to further improve the performance of MC-CDMA system [15]. To further enhance the performance of MC-CDMA system, in this work B-STTC based STBC site diversity is proposed and analysed. The use of B-STTC in STTC based STBC reduces the error rate and pave way improvement in performance. Furthermore, it is extended to STBC based B-STTC to attain site diversity.

\section{B-STTC Based STBC Site Diversity Technique for MC-CDMA System}

B-STTC based STBC site diversity is proposed to improve the performance of MC-CDMA system in multi-path fading environment. B-STTC based STBC code exploit diversity gain and channel efficiency simultaneously without aggravating a bandwidth expansion similar to STTC based STBC codes, nevertheless with decreased error rate in view of the fact that B-STTC is used in replacement to STTC block.

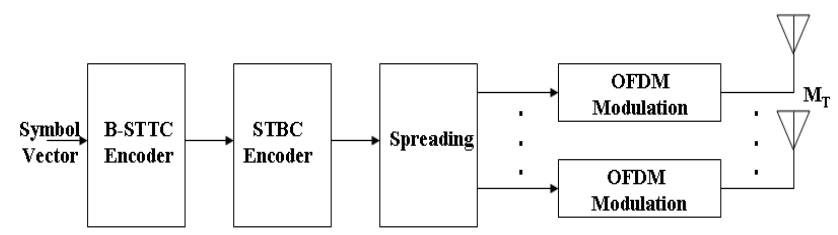

Figure 1. Block diagram of B-STTC based STBC site diversity transmitter for MC-CDMA system

Figure 1 represents the block diagram of B-STTC based STBC site diversity transmitter for MC-CDMA system. The input symbol vectors are modulated by a B-STTC and STBC encoder and transmitted as a conventional MC-CDMA system. The receiver model provided for STBC site diversity [8] can be used here as it involves normal STBC decoding operation.

In the transmitter, input bits are fed to the encoder with the help of memoryless source $S_{R}=\{0,1\}$ with equally probable symbols [11], the modulation for a given state is denoted as

$$
X=\left(x_{1} x_{2}, \ldots, x_{u}\right)^{T} \in \mathbb{Z}_{4}^{i_{T}}
$$

where $\mathbb{Z}_{4}^{i_{T}}$ represents the index of Q-PSK symbols at transmitting antenna of the shift register realized by $(v+1)$ blocks of $u$ bits and $x_{1} x_{2}, \ldots, x_{u}$ are the modulated symbols. The B-STTC generated by the STTC encoder with Q-PSK is given by

$$
F=S_{B} X
$$

where $S_{B}$ is the generator matrix given by

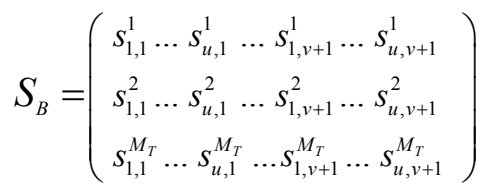

For three transmit and receive antenna case, the encoder with a help of three constellation symbols $s_{1}, s_{2}$ and $s_{3}$ generates blocks $s_{1}, s_{2}, s_{3},-s_{1}^{*}, s_{2}^{*}$ and $-s_{3}^{*}$. Signal $s_{1}$ is transmitted from first antenna, $s_{2}$ is transmitted from second antenna and $s_{3}$ is transmitted from third antenna at time $t=1$. Signals $-s_{1}^{*}, s_{2}^{*}$ and $-s_{3}^{*}$ are transmitted from first, second and third antenna respectively at time $t=2$.

Assuming that the fading remains constant, the received signals from three different antennas are denoted as

$$
\begin{aligned}
& y_{1}=h_{1} s_{1}+h_{2} s_{2}+h_{3} s_{3}+n_{1} \\
& y_{2}=-h_{1} s_{2}^{*}+h_{2} s_{2}^{*}-h_{3} s_{3}^{*}+n_{2} \\
& y_{3}=h_{1} s_{3}-h_{2} s_{3}^{*}+h_{3} s_{3}+n_{3}
\end{aligned}
$$

Where $h_{1}, h_{2}$ and $h_{3}$ are the path gains modelled as independent samples of a zero mean complex Gaussian random variable $n_{1}, n_{2}$ and $n_{3}$ are random Gaussian variables with zero mean and variance $\sigma^{2}$.

The receiver generates soft estimates with the assistance of perfect channel state information (CSI) [10] as follows

$$
\begin{aligned}
\tilde{s}_{1} & =h_{1}^{*} y_{1}+h_{2} y_{2}+h_{3} y_{3}^{*} \\
& =\left(\left|h_{1}\right|^{2}+\left|h_{2}\right|^{2}+\left|h_{3}\right|^{2}\right) s_{2}+h_{1}^{*} n_{1}+h_{2} n_{2}+h_{3} n_{3}^{*} \\
\tilde{s}_{2} & =h_{2} y_{1}+h_{1} y_{2}^{*}+h_{3}^{*} y_{3} \\
& =\left(\left|h_{1}\right|^{2}+\left|h_{2}\right|^{2}+\left|h_{3}\right|^{2}\right) s_{3}+h_{2} n_{1}+h_{1} n_{2}^{*}+h_{3}^{*} n_{3} \\
\tilde{s}_{3}= & h_{3} y_{1}^{*}+h_{2} y_{2}+h_{3}^{*} y_{3} \\
= & \left(\left|h_{1}\right|^{2}+\left|h_{2}\right|^{2}+\left|h_{3}\right|^{2}\right) s_{1}+h_{3} n_{1}^{*}+h_{2} n_{2}+h_{3}^{*} n_{3}
\end{aligned}
$$

With the first transmitted symbol $s_{1}$, the Viterbi decoder builds the following metric for the branch symbol $s_{M_{R}}$, as given below 


$$
\begin{aligned}
& m_{e}\left(\tilde{s}_{1}, s_{M_{R}}\right)=\left(\left|h_{1}\right|^{2}+\left|h_{2}\right|^{2}+\left|h_{3}\right|^{2}\right)\left|s_{M_{R}}\right|^{2}+d_{e}^{2}\left(\tilde{s}_{1}, s_{M_{R}}\right) \\
& m_{e}\left(\tilde{s}_{2}, s_{M_{R}}\right)=\left(\left|h_{1}\right|^{2}+\left|h_{2}\right|^{2}+\left|h_{3}\right|^{2}\right)\left|s_{M_{R}}\right|^{2}+d_{e}^{2}\left(\tilde{s}_{2}, s_{M_{R}}\right) \\
& m_{e}\left(\tilde{s}_{3}, s_{M_{R}}\right)=\left(\left|h_{1}\right|^{2}+\left|h_{2}\right|^{2}+\left|h_{3}\right|^{2}\right)\left|s_{M_{R}}\right|^{2}+d_{e}^{2}\left(\tilde{s}_{3}, s_{M_{R}}\right)
\end{aligned}
$$

Where $m_{e}$ denotes the metric of the soft estimates associated with $s_{M_{R}} \quad d_{e}^{2}\left(\tilde{s}_{1}, s_{M_{R}}\right), d_{e}^{2}\left(\tilde{s}_{2}, s_{M_{R}}\right)$ and $d_{e}^{2}\left(\tilde{s}_{3}, s_{M_{R}}\right)$ are the Euclidean distance obtained from $\left(\tilde{s}_{1}, s_{M_{R}}\right)\left(\tilde{s}_{1}, s_{M_{R}}\right)^{*} \quad, \quad\left(\tilde{s}_{2}, s_{M_{R}}\right)\left(\tilde{s}_{2}, s_{M_{R}}\right)^{*} \quad$ and $\left(\tilde{s}_{3}, s_{M_{R}}\right)\left(\tilde{s}_{3}, s_{M_{R}}\right)^{*}$ respectively.

Performance of the system under fading channel remains constant for two consecutive symbol transmission periods, $2 T_{p}$. Analysis of the system is carried out through the decoding data $E$ obtained from the maximum likelihood decoder in the receiver when the coded sequence $S$ is transmitted. $S$ and $E$ are defined as

$$
\begin{aligned}
& S=\left[s_{1}, s_{2},-s_{2}^{*}, s_{1}^{*}, \ldots, S_{2 T_{p}-1},-s_{2 T_{p}-1}^{*}, s_{2 T_{p}-2}^{*}\right] \\
& E=\left[e_{1}, e_{2},-e_{2}^{*}, e_{1}^{*}, \ldots, e_{2 T_{p}-1},-e_{2 T_{p}-1}^{*}, e_{2 T_{p}-2}^{*}\right]
\end{aligned}
$$

where $e_{1}$ and $e_{2}$ are the decoded data of the symbols $S_{1}$ and $S_{2}$

The error probability approximation is obtained as

$$
P_{e}\left(S \rightarrow E \mid h_{1}, h_{2}\right)=\exp \left\{-\frac{E_{s}}{4 \sigma^{2}} \sum_{r_{x}=1,2}^{2 T_{p}-2}\left\{\left[\mid\left[s_{l}-\left.e_{l}\right|^{2}+\right]\left[\left|h_{l}-e_{l+1}\right|^{2}+\left|h_{2}\right|^{2}\right]\right\}\right\}\right.
$$

Where

$l$ is the symbol duration

$T_{p}$ is the symbol transmission period

$E_{s}$ is the average energy of the signal constellation

For an independent Rayleigh fading distribution of $r_{a} M_{R}$ and $\left|h_{1}\right|$ with probability density of

$$
\begin{gathered}
P\left(\left|h_{1}\right|\right)=2\left|h_{1}\right| \exp \left(-\left|h_{1}\right|^{2}\right) \\
P_{e}(S \rightarrow E) \leq E \prod_{l=1,2}^{2 T_{p}-2} \exp \left\{-\frac{E_{s}}{4 \sigma^{2}}\left[\left|s_{l}-e_{l}\right|^{2}+\left|s_{l}-e_{l+1}\right|^{2}\right]\left[\left|h_{1}\right|^{2}+\left|h_{2}\right|^{2}\right]\right\} \\
=\prod_{l=1}^{2 T_{p}-2} E_{O}\left(\exp \left\{-\frac{E_{s}}{4 \sigma^{2}}\left[\left|s_{l}-e_{l}\right|^{2}+\left|s_{l}-e_{l+1}\right|^{2}\right]\left|h_{1, M_{R}}\right|^{2}\right\}\right)
\end{gathered}
$$

With the aid of equation (8), the error event probability, the coded sequence $S$ is decided to $E$ is expressed as

$$
\begin{aligned}
P_{e}(S \rightarrow E) & \leq\left(\prod_{l=1}^{r_{a}}\left[\left|s_{l}-e_{l}\right|^{2}+\left|s_{l}-e_{l+1}\right|^{2}\right]\right)^{-M_{R}}\left(\frac{E_{s}}{4 \sigma^{2}}\right)^{-r_{a} M_{R}} \\
& =\left(\prod_{l=1}^{r_{a}}\left[\left|s_{l}-e_{l}\right|^{2}+\left|s_{l}-e_{l+1}\right|^{2}\right]^{1 / r_{a}}\right)^{-r_{a} M_{R}}\left(\frac{E_{s}}{4 \sigma^{2}}\right)^{-r_{a} M_{R}}
\end{aligned}
$$

where

$r_{a}$ is the rank of the coded matrix

$r_{a} M_{R}$ is the diversity gain

$\prod_{l=1}^{r_{a}}\left[\left|c_{l}-e_{l}\right|^{2}+\left|c_{l}-e_{l+1}\right|^{2}\right]^{1 / r_{a}}$ is the coding gain

By this method B-STTC based STBC site diversity technique system is realised for the system which obtains coding gain without provoking a bandwidth expansion.

\section{STBC Based B-STTC Site Diversity Technique for MC-CDMA System}

Figure 2 depicts the block diagram of STBC based B-STTC site diversity transmitter for MC-CDMA system and the receiver can utilise STTC site diversity [10] technique. STBC based B-STTC codes are similar to STBC based STTC codes with balanced constellation points in STTC block. Like STBC based STTC codes, STBC based B-STTC codes are also built by set partitioning. The goal of set partitioning is to achieve a better coding gain and is obtained through pair-wise distance. As STBC based B-STTC codes have equally probable constellation points at the decoder it reduces the error rate compared to B-STTC based STBC codes.

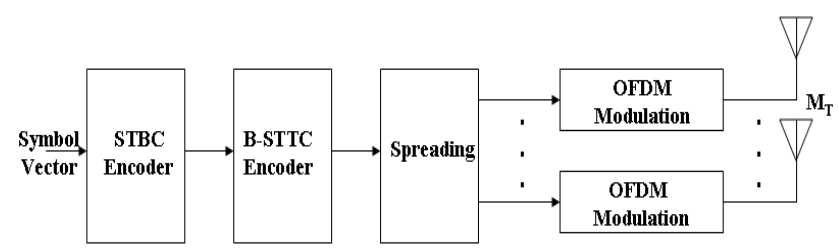

Figure 2. Block diagram of STBC based B-STTC site diversity transmitter for MC-CDMA

For STBC codes, the determinant criterion defines the pair-wise distance which is used to establish the partitioning rules. If the transmission matrix of space time code is denoted as $c_{1}=G\left(s_{1}, s_{2}\right), D_{f}\left(c_{1}, c_{2}\right)=G\left(s_{1}, s_{2}\right)-G\left(s_{1}{ }^{\prime}, s_{2}{ }^{\prime}\right)$ represents the difference of the transmission matrices for codewords $c_{1}$ and $c_{2}$, the diversity is defined by the minimum rank of the matrix $D_{f}\left(c_{1}, c_{2}\right)$. The minimum of the determinant of the matrix $B\left(c_{1}, c_{2}\right)=D_{f}^{H}\left(c_{1}, c_{2}\right) D_{f}\left(c_{1}, c_{2}\right)$, where $D_{f}^{H}\left(c_{1}, c_{2}\right)$ is the Hermition difference of the transmission matrix, over all possible pairs of distinct codewords $c_{1}$ and $c_{2}$ [16] corresponds to the coding gain. Using this definition CGD between codewords $\left(c_{1}, c_{2}\right)$ is given as

$$
C G D\left(c_{1}, c_{2}\right)=D_{f}^{2}\left(c_{1}, c_{2}\right)=\operatorname{det}\left(B\left(c_{1}, c_{2}\right)\right)
$$

where 


$$
\begin{gathered}
B\left(c_{1}, c_{2}\right)=\left[\begin{array}{ccc}
2\left|z_{1}\right|^{2} & -\left(z_{2}\right)\left(z_{1}\right)^{*} e^{j \beta} & \left(z_{2}\right)^{*}\left(z_{1}\right) \\
-\left(z_{3}\right)^{*}\left(z_{2}\right) e^{-j \beta} & \left|z_{2}\right|^{2}+\left|z_{3}\right|^{2} & \left(z_{2}\right)^{*}\left(z_{1}\right) e^{-j \alpha} \\
\left(z_{3}\right)\left(z_{1}\right)^{*} & \left(z_{3}\right)^{*}\left(z_{1}\right) e^{j \alpha} & 2\left|z_{3}\right|^{2}
\end{array}\right] \\
z_{1}=s_{1}-s_{1}{ }^{\prime} ; z_{2}=s_{2}-s_{2}{ }^{\prime} ; z_{3}=s_{3}-s_{3}{ }^{\prime}
\end{gathered}
$$

The STBC orthogonal design coupled via symbol $s_{2}$ for three transmit and receive antennas is denoted as

$$
G\left(s_{1}, s_{2}, s_{3}\right)=\left[\begin{array}{ccc}
-s_{2}{ }^{*} & s_{1} & s_{1}{ }^{* j \beta} \\
x_{1}{ }^{*} & s_{2} & -s_{3}{ }^{*} \\
s_{3}{ }^{*} e^{j \alpha} & s_{3} & s_{2}{ }^{*}
\end{array}\right]
$$

where $s_{1}{ }^{*} e^{j \beta}$ and $s_{3}{ }^{*} e^{j \alpha}$ enable to optimise the minimum CGD within each obtained coset, angles $(\alpha, \beta)$ belong to the constellation symbol and are used to separate the triplets of symbols which share the same second symbol.

The set partitioning for Q-PSK constellation is obtained through the mathematical expression of

$$
\operatorname{det}\left(B\left(c_{1}, c_{2}\right)\right)=\left|z_{1}\right|^{4}\left|z_{3}\right|^{2}+\left|z_{1}\right|^{2}\left|z_{3}\right|^{4}-2 \operatorname{Re}\left[\left(z_{3}^{*}\right)^{3}\left(z_{1}\right)^{3} e^{j(\alpha-\beta)}\right]
$$

The choice of $(\alpha, \beta)$, which maximises the minimum CGD within each coset, is sampled at a rate $\pi / 16$. However, the obtained values for $(\alpha, \beta)$ does not correspond to Q-PSK constellation points [17] and this necessitates the need for constellation expansion to use set partitioning with maximum of minimum CGD values. Applying set partitioning for 16 cosets gives a minimum CGD values of 128 and without constellation expansion [18], using set partitioning for the same number of cosets gives minimum CGD of 8 with $(\alpha, \beta)=(0,0)$.

A simple STBC structure is used to derive a set partitioning rule. This structure will be a basis to obtain a powerful quasi orthogonal structure which will be incorporated into a trellis. The STBC matrix looks like

$$
G\left(s_{1}, S_{2}, S_{3}\right)=\left[\begin{array}{ccc}
-S_{2}^{*} & s_{1} & 0 \\
s_{1}^{*} & s_{2} & -S_{3}^{*} \\
0 & S_{3} & s_{2}^{*}
\end{array}\right]
$$

The computation of the determinant of matrix $B\left(c_{1}, c_{2}\right)$ with $_{s_{1}}=e^{j p_{1} \omega}$,

$$
\begin{gathered}
s_{2}=e^{j p_{2} \omega}, s_{3}=e^{j p_{3} \omega} \text { and } s_{1}{ }^{\prime}=e^{j q_{1} \omega}, \\
s_{2}{ }^{\prime}=e^{j q_{2} \omega}, s_{3}{ }^{\prime}=e^{j q_{3} \omega}
\end{gathered}
$$

where $p_{1}, p_{2}, p_{3}, q_{1}, q_{2}, q_{3}$ are the integers yields the following expression

$$
\begin{aligned}
& \operatorname{det}\left(B\left(c_{1}, c_{2}\right)=64 \sin ^{2}\left(\frac{\left|q_{2}-p_{2}\right|}{2} \omega\right)\right. \\
& \left(\sin ^{2}\left(\frac{\left|q_{1}-p_{1}\right|}{2} \omega\right)+\sin ^{2}\left(\frac{\left|q_{2}-p_{2}\right|}{2} \omega\right)+\sin ^{2}\left(\frac{\left|q_{3}-p_{3}\right|}{2} \omega\right)\right)^{2}
\end{aligned}
$$

where $\omega=2 \pi / P_{c} \quad\left(P_{c}\right.$ is the size of the transmit constellation) and $\omega=\pi / 2$ for a Q-PSK constellation. Equation (15) clearly implies that symbols $s_{1}$ and $s_{1}^{\prime} ; s_{2}$ and $s_{2}^{\prime} ; s_{3}$ and $s_{3}^{\prime}$ have to be different in each coset to maintain a non-null CGD.

After the set partitioning step, a trellis is built affecting a particular STBC from a set of possible candidates to transitions originating from a state. The coding gain is optimised within each coset by maximising the distance between codewords. A design with full diversity is needed to use STBC code given in equation (14) into a STTC design and in fact, achieving full diversity is equivalent to showing the determinants of matrices $B\left(c_{1}, c_{2}\right)$ are nonzero over all possible codewords $c_{1}$ and $c_{2}$. As it is the case, matrices $B\left(c_{1}, c_{2}\right)$ should be checked that they do not loose the full rank value when $c_{1}$ and $c_{2}$ belong to different cosets. To solve this unit transform matrices $\Theta$ are assigned to each state to check that the minimum of $\operatorname{det}\left(B\left(c_{1}, c_{2}\right)\right)$ is equal to zero when $c_{1}$ belongs to coset 1 and $c_{2}$ belongs to coset 2 . In fact unitary matrix $\Theta_{\mathrm{j}}$ corresponds to a rotation and preserves distance among the constellation points i.e., the minimum CGD value is left unchanged by applying a unitary transform $\Theta_{\mathrm{j}}$ to $G\left(s_{1}, s_{2}, s_{3}\right)$ within each coset. Search for unitary matrices are done using parameterisation [19], and along with the selected partitioning level, unit matrices whose number equal to the number of cosets with maximum separation distance are obtained. The trellis is evenly built by affecting a unitary matrix transform to each coset with each of them corresponding to a trellis state and is transmitted. Assuming Rayleigh fading channel, received signal within three successive time slot intervals, is given by

$$
\begin{aligned}
& y_{1}=-h_{1} s_{2}^{*}+h_{2} s_{1}^{*}+h_{3} s_{3}^{*} e^{j \alpha}+n_{1} \\
& y_{2}=h_{1} s_{1}+h_{2} s_{2}+h_{3} s_{3}+n_{2} \\
& y_{3}=h_{1} s_{1}^{*} e^{j \beta}-h_{2} s_{3}^{*}+h_{3} s_{2}^{*}+n_{3}
\end{aligned}
$$

To compute branch metrics for different symbol triplets, auxiliary quantities are obtained as below

$$
\begin{aligned}
& y_{1}^{\prime}=y_{1}-h_{3} s_{3}^{*} e^{j \alpha} \\
& y_{2}^{\prime}=y_{2}-h_{3} s_{3} \\
& y_{2}^{\prime \prime}=y_{2}-h_{1} s_{1} \\
& y_{3}^{\prime}=y_{3}-h_{1} s_{1}^{*} e^{j \beta}
\end{aligned}
$$

The decoding signal is obtained through combining [20] with equations (16) and (17) and is given by

$$
\begin{aligned}
& y_{1}^{*} h_{2}^{j}+h_{1}^{*} y_{2}^{\prime}=\left(\left|h_{1}\right|^{2}+\left|h_{2}\right|^{2}\right) s_{1}+n_{2} h_{1}^{*}+n_{1}^{*} h_{2} \\
& -y_{1}^{*} h_{1}^{j}+h_{2}^{*} y_{2}^{\prime}=\left(\left|h_{1}\right|^{2}+\left|h_{2}\right|^{2}\right) s_{1}+n_{2} h_{2}^{*}-n_{1}^{*} h_{1} \\
& y_{3}^{*} h_{3}+h_{2}^{*} y_{2}^{\prime \prime}=\left(\left|h_{2}\right|^{2}+\left|h_{3}\right|^{2}\right) s_{2}+n_{2} h_{2}^{*}+n_{3}^{*} h_{3} \\
& -y_{3}^{*} h_{2}+h_{3}^{*} y_{2}^{\prime \prime}=\left(\left|h_{2}\right|^{2}+\left|h_{3}\right|^{2}\right) s_{3}+n_{2} h_{3}^{*}-n_{3}^{*} h_{2}
\end{aligned}
$$




\section{Simulation Results}

The proposed site diversity for MC-CDMA system is simulated using MATLAB and the simulation parameters are given in Table 1. Figure 3 shows the symbol error rate (SER) performance with respect to energy per bits to the spectral noise density $\left(E_{b} / N_{0}\right)$ of the system with and without diversity under Rayleigh fading channel. The diversity technique uses two antennas at the transmitter and receiver terminal. The result indicates that when diversity was used there is an improvement in BER performance of the system due to the exploitation of multiple antennas in the transmitter and receiver.

Table 1.. Simulation Parameters

\begin{tabular}{|c|c|}
\hline Parameters & Values \\
\hline Modulation & 4-PSK \\
\hline Symbol length & 64 \\
\hline No.of sub- carriers & 128 \\
\hline Channel estimation & Perfect estimation \\
\hline Channelisation code & Walsh-Hadamard code of length 63 \\
\hline Scrambling code & Random code of length 63 \\
\hline Channel & $\begin{array}{c}\text { Rayleigh fading channel with AWGN } \\
\text { floor }\end{array}$ \\
\hline
\end{tabular}

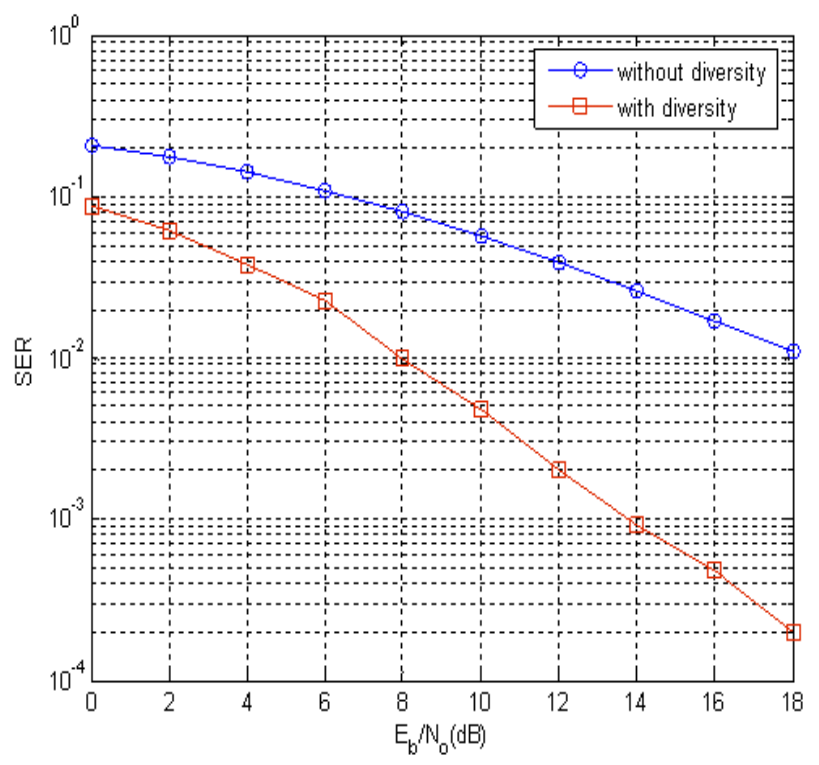

Figure 3. Performance of the system with and without diversity

Performance of MC-CDMA system with STBC based STTC site diversity is evaluated by varying the transmitting and receiving antennas. Figure 4 illustrates the performance of the system in terms of SER and $\mathrm{E}_{\mathrm{b}} / \mathrm{N}_{0}$ with STBC based STTC site diversity for two, three, four and five transmit and receive antennas. It is observed from the plots that the system with five transmit and receive antennas gives better performance in SER when compared to the system with two, three and four antennas. The improvement in SER for the larger number of antennas is due to the maximum utilisation of diversity. The maximum number of antennas used for simulation is restricted to five as further increase of it introduces hardware complexity and increases the cost of the system.

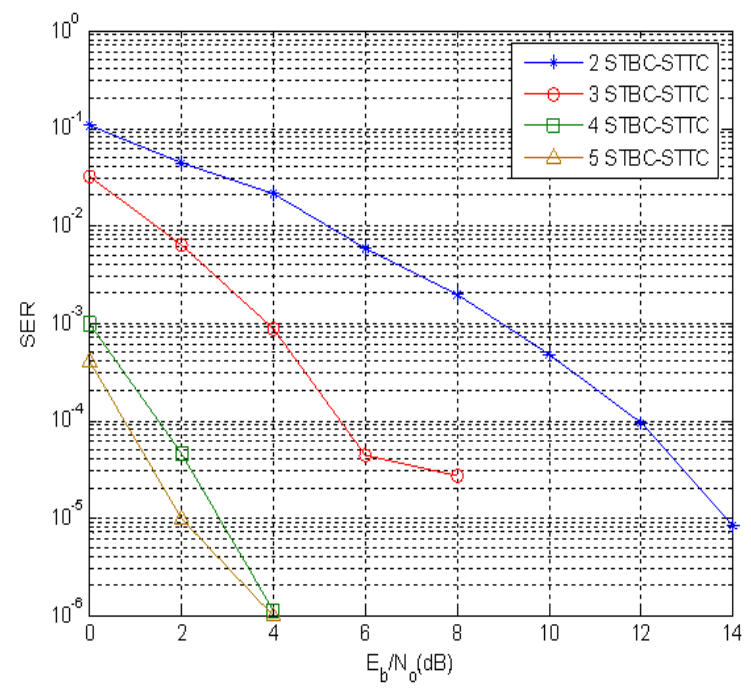

Figure 4. Performance of the system with STBC based STTC site diversity for various antennas

Figure 5 depicts $\mathrm{SER}$ versus $\mathrm{E}_{\mathrm{b}} / \mathrm{N}_{0}$ performance of the system with B-STTC based STBC site diversity for various numbers of antennas. The result portrays that the SER of the system with five transmit and receive antennas outperforms two, three and four transmit and receive antennas. Figure 6 renders the same scenario of the system with STBC based B-STTC site diversity for different antennas. Similar conditions of diversity utilisation observed in Figures 4 and 5 are noticed here irrespective of the coding techniques used for site diversity technique i.e. improvement of SER is noticed clearly when there is increase in number of antennas.

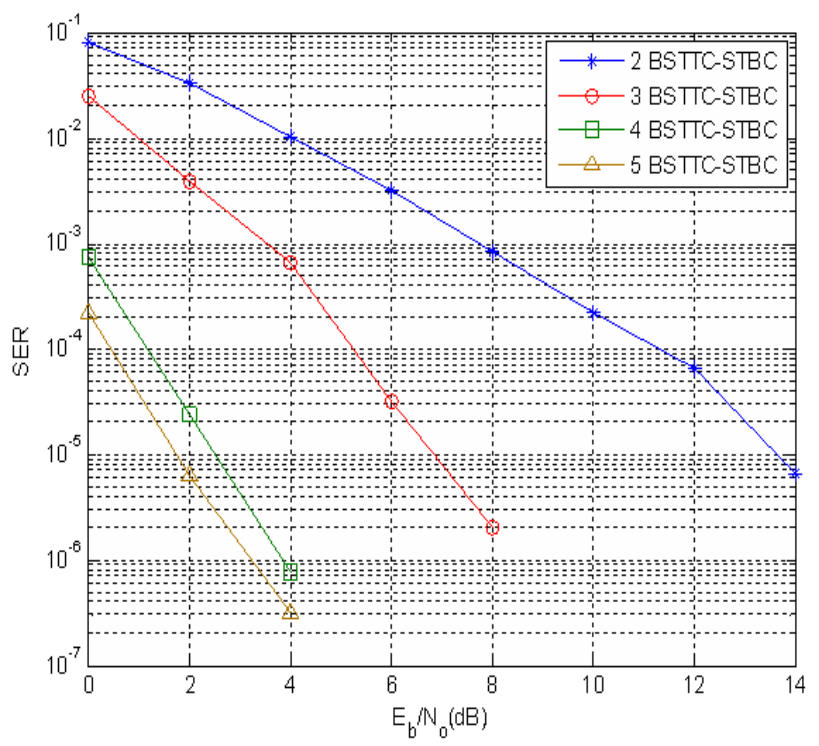

Figure 5. Performance of the system with B-STTC based STBC site diversity for various antennas 


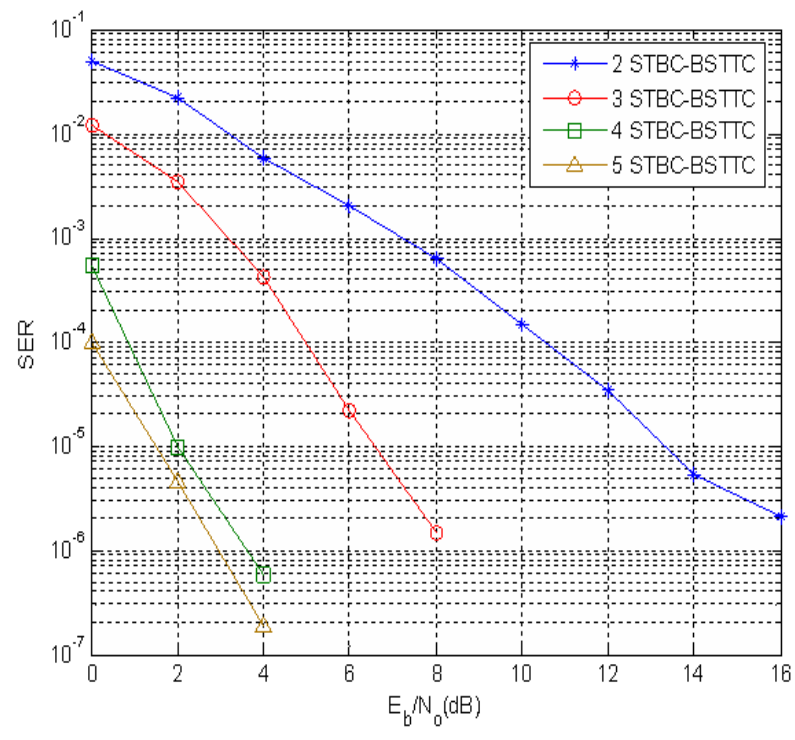

Figure 6. Performance of the system with STBC based B-STTC site diversity for various antennas

The SER performance of STBC based STTC site diversity is compared with the B-STTC based STBC site diversity in Figure 7. It is clearly visible from this figure that B-STTC based STBC site diversity technique outshines STBC based STTC site diversity technique of the system. B-STTC based STBC site diversity uses balanced codes in STTC encoder block which reduces the error rate when compared to STBC based STTC site diversity technique.

SER performance of the system is compared between B-STTC based STBC site diversity technique and STBC based B-STTC site diversity technique and is depicted in Figure 8. This figure clearly portrays that STBC based B-STTC site diversity technique surpasses B-STTC based STBC site diversity technique. As the STBC based B-STTC codes are transmitted in STTC form and moreover in balanced manner (equal probability of constellation points), the improvement in error performance occurs.

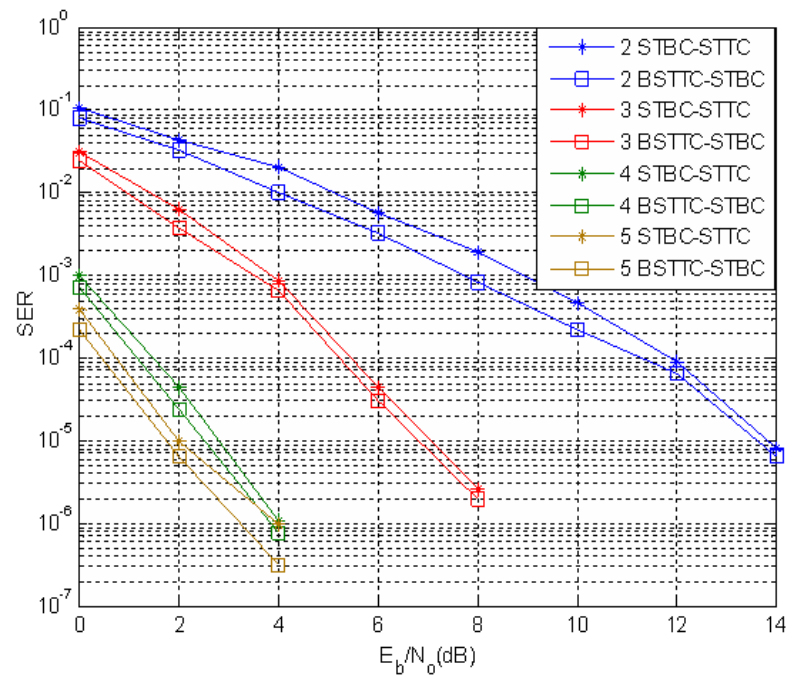

Figure 7. Performance comparison of the system with STBC based STTC and B-STTC based STBC site diversity techniques for various antennas

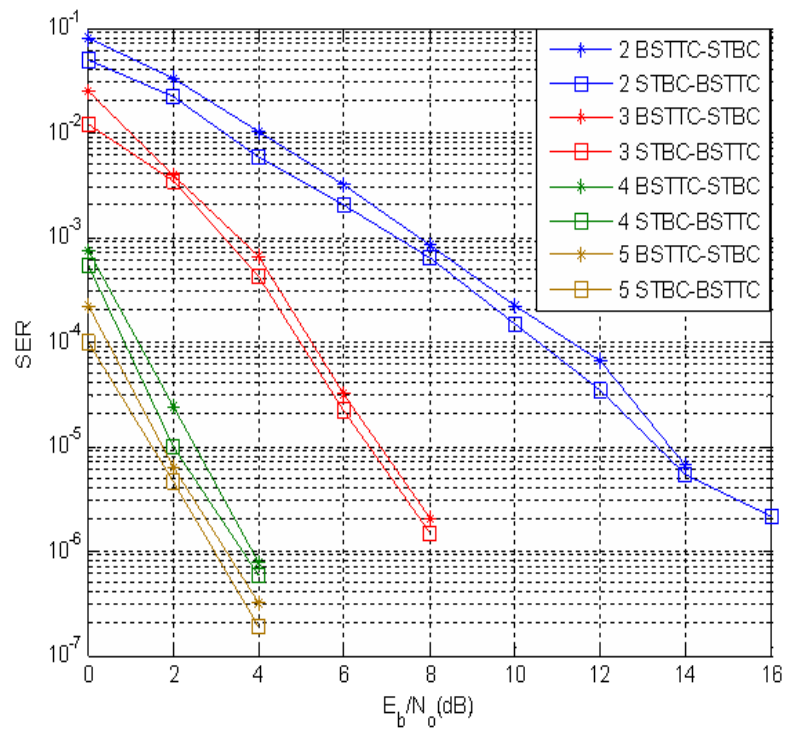

Figure 8. Performance comparison of the system with STBC based STTC and B-STTC based STBC site diversity techniques for various antennas

\section{Conclusion}

In this paper, site diversity scheme for MC-CDMA system is proposed using B-STTC based STBC and STBC based B-STTC to improve the performance of mobile terminals in the downlink. These methods considerably minimise multi-cell interference by jointly consuming the diversity gain and coding gain. With STBC based STTC site diversity technique, the performance of MC-CDMA system achieves better reduction in error rates with additional diversity gain as it is transmitted in the form of B-STTC codes. Simulation results shows that STBC based B-STTC site diversity outperforms STBC based STTC and B-STTC based STBC site diversity techniques in terms of SER and is best opted for MC-CDMA system. Further, irrespective of the coding techniques used for site diversity, improvement of SER is noticed clearly when there is increase in number of antennas. This clearly indicates the maximum utilisation of diversity with increased number of antennas. In near future space time codes with layered architecture can be engaged to improve the system performance along with spatial multiplexing technique.

\section{REFERENCES}

[1] Hara S, Prasad R. Design and performance of multicarrier CDMA system in frequency selective Rayleigh fading channels. IEEE Transactions on Vehicular Technology Vol.48, No.5, 1584-1595.

[2] Hara S, Prasad R., Overview of multi carrier CDMA. IEEE Communication Magazine, Vol. 35, No.12, 126-133.

[3] Chatterjee S, Fernando WAC, Wasantha MK. Adaptive 
modulation based MC-CDMA systems for $4 \mathrm{G}$ wireless consumer applications. IEEE Transactions on Consumer Electronics, Vol.49, No.4, 995-1003.

[4] Foschini G, Gans M. On the limits of wireless communications in a fading environment when using multiple antenna. Wireless Personal Commuication, Vol. 6, No.3, 311-335.

[5] Alamouti SM. A simple transmit diversity technique for wireless communications. IEEE Journal on Selected Areas in Communication, Vol. 16, No.8, 1451-1458.

[6] Inoue M, Fujii T, Nakagawa M. Space time transmit site diversity for OFDM multi- base station system. International Workshop on Mobile and Wireless Communications Network, Sweden, 30-34, 2002.

[7] Wong D, Lim TJ. Soft handoffs in CDMA mobile systems. IEEE Personal Communications, Vol. 36, No.6, 6-17.

[8] Kumarathran N, Padmavathy M, Dananjayan P. Performance improvement of MC-CDMA system through STBC site diversity. International Journal of Emerging Technologies and Applications in Engineering, Technologies and Sciences, Vol. 1, No.1, 1-7.

[9] Tarokh V, Seshadri N, Calderbank AR. Space-time codes for high data rate wireless communication: Performance criterion and code construction. IEE Transactions on Information Theory, Vol. 44, No.2, 744-765.

[10] Kumarathran N, Jayapriya S, Dananjayan P. STTC based site diversity technique for MC-CDMA System. IEEE International Conference on Control, Automation, Communication and Energy Conservation, India, 2: 695-700, 2009.

[11] Ngo TMH, Zaharia G, Bougeard S, Helard JF. Design of balanced QPSK space- time trellis codes for several transmit antennas. International Symposium on Signals, Circuits and Systems, Lasi, 2: 1-4, 2007.
[12] Kumarathran N, S.Jayapriya S, Dananjayan P. Performance improvement of MC-CDMA system through B-STTC site diversity. International Journal of Wireless and Mobile Communication. (Communicated)

[13] Ferre G, Cances JP, Meghdadi V, Dumas JM. STBC based STTC codes optimized designs for three transmit antennas. Special issue on Communication Systems, Networks and Digital Signal Processing, Vol. 3, No. 3, 110-118.

[14] Janani M, Nosratinia A. Generalized block space-time trellis codes: Set-partitioning and code design. IEEE Wireless Communication and Networking Conference, Sweden, 461-465, 2005.

[15] Kumarathran N, Jayapriya S, Dananjayan P. Performance Enhancement of MC-CDMA System through STBC based STTC Site Diversity. International Journal of Computer and Electrical Engineering Vol. 2, No.1, 6-11.

[16] Ferre G, Cances J, Meghdadi V, and Dumas J. STBC based (turbo) STTC codes built by set partitioning for three transmit antennas: construction and performances. IEEE Transactions on Wireless Communication, Vol. 6, No.3, 827-832.

[17] Chen Z, Vucentic B, Yuan J, Lo K. Space-time trellis coded modulation with three and four antennas on slow fading channels. IEEE Communication Letters, Vol. 6, No.2, 67-69.

[18] Chen Z, Yuan J, Vucetic B. Improved space-time trellis coded modulation scheme on slow Rayleigh fading channels. IEEE Electronics Letters, Vol.37, No.7, 440-451.

[19] Jing Y, Hassibi B. Three transmit antenna space-time codes based based on SU(3). IEEE Transactions on Signal Processing, Vol. 53, No. 10, 3688-3702.

[20] Eng T, Kong N, Milstein LB. Comparison of diversity combining techniques for Rayleigh fading channels. IEEE Transactions on Communications, Vol. 44, No.9, 1117-1129. 